\title{
NOTE COMPLEMENTAIRE SUR LES ECHINODERMES ORDOVICIENS DE SIERRA MORENA
}

\author{
J. Chauvel $\left({ }^{*}\right)$ et B. Meléndez $\left({ }^{* *}\right)$
}

\section{RÉSUMÉ}

Une nouvelle espèce du genre Calix est décrite, le genre Batalleria est redécrit, la présence de Phlyctocystis dans les Monts de Tolède-Sierra Morena est confirmée, et celle de Destombesia est signalée.

Mots clés: Sierra Morena, Ordovicien, Cystoides, Systématique, Biestratigraphie.

\begin{abstract}
Addendum on the Ordovician Cystoids of Sierra Morena. A number of specimens from the synclinorium of Almaden (Ciudad Real), have allowed the description of a new species of the genus Calix, and a more complete description of the genus Batalleria. Also, it is confirmed the presence there of genus Phlyctocystis, as well as the first finding of the genus Destombesia in this area.
\end{abstract}

Key words: Sierra Morena, Ordovician, Cystoids, Systematics, Biostratigraphy.

\section{RESUMEN}

Se describe una nueva especie del género Calix del Sinclinorio de Almadén; se presenta una nueva descripción del género Batalleria a base de nuevos ejemplares ahora estudiados; se confirma la presencia de Phlyctocystis en la provincia de Ciudad Real y se señala, por primera vez, el género Destombesia.

Palabras clave: Sierra Morena, Ordovicico, Cistideos, sistemática, biostratigrafía.

\section{Introduction}

Les Monts de Tolède et la Sierra Morena ont révélé une faune d'Echinodermes assez variée; elle a fait l'objet de plusieurs publications, dont une récente (Chauvel et Meléndez in Gutiérrez Marco et al., 1984), qui peut être completée grâce à de nouvelles trouvailles. Les gissements concernés appartiennent tous à l'Ordovicien moyen ou supérieur, ce sont:

Localité AL-IV (Synclinal d'Almadén). Km 24 de la route C-424 d'Almadén à Puertollano (C.R.); correspondant au tiers inférieur des "schistes Guindo". L'âge des niveaux fossilifêres est le Llandeilo, partie supérieure de la zone à Placoparia (Coplacoparia) tournemini (subzone à Morgatia hupei).

Localité CC-IV (Calzada de Calatrava, C.R.). Situé aux alentours de la route C-410 d'Almuradiel à Calzada de Calatrava (C.R.), en bas du barrage du fleuve Fresneda. Même possition stratigraphique et âge de la localité précédente.

Localité VM-III (Viso del Marqués, C.R.). Quelque $1.000 \mathrm{~m}$ en direction $\mathrm{N}-145^{\circ} \mathrm{W}$ du sommet géodesique "Palacio de la Marina", Même possition stratigraphique et âge de la localité précédente.

Localité AL-VII (Synclinal d'Almadén). Km 11 de la route d'Almadén à Saceruela (C.R.), dans la rive

(*) Université de Rennes. Institut de Géologie. Avenue du Géneral Leclerc, 35042 Rennes Cedex, France.

(**) Departamento de Paleontología. Facultad de Ciencias Geológicas. Universidad Complutense. 28040 Madrid. 

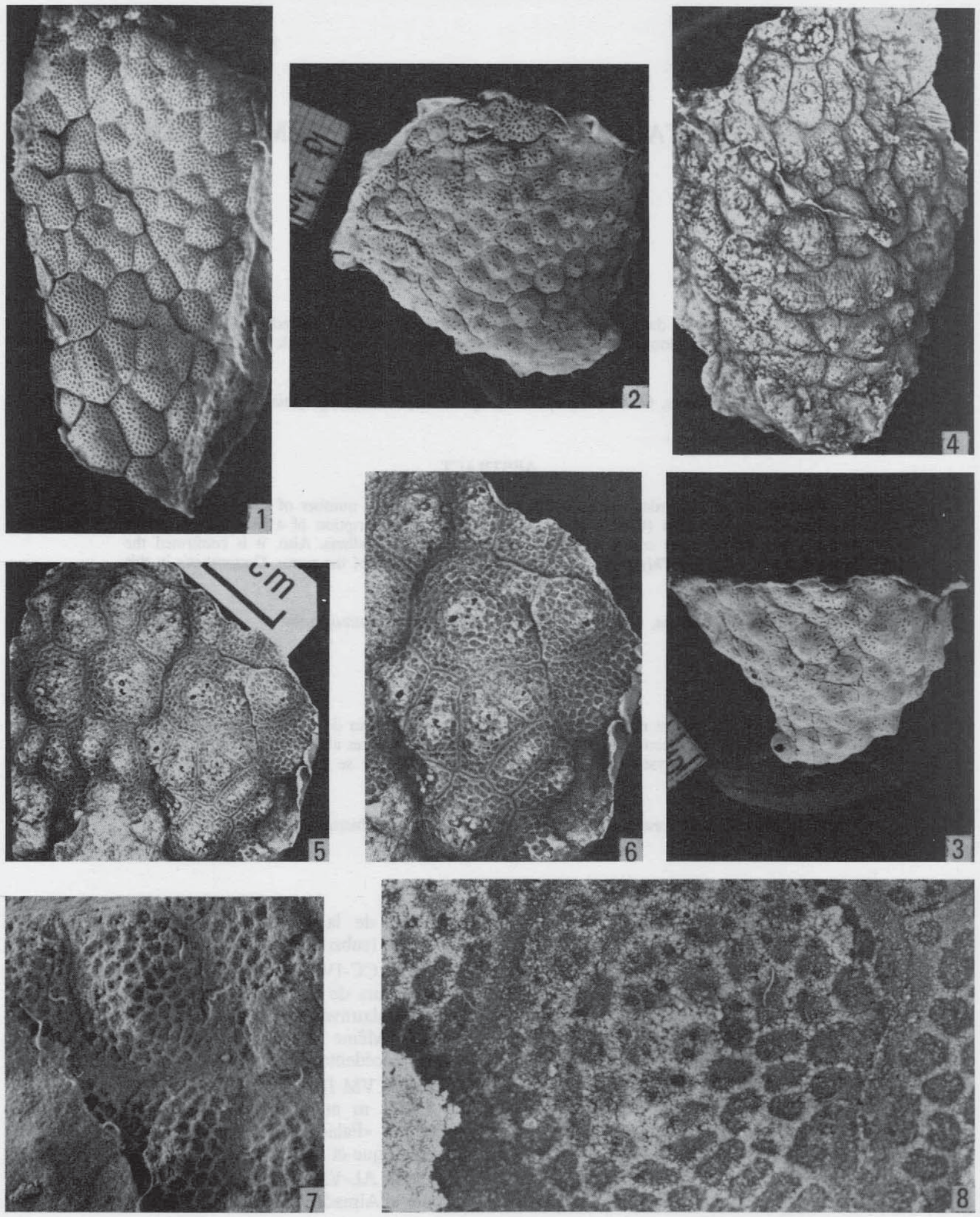

Planche I.-Fig. 1: Phlyctocystis granulata; MT-209, moulage en latex (x 1). Figs. 2-3: Calix sp.: 2, MT-213, moulage en latex, vue latérale de la face convexe (x 1,5); 3, même exemplaire, vue de l'autre face (x 1,5). Figs. 4-8: Calix gutierrezi: 4, MT-224, moulage en latex (x 1,5); 5, MT-226, moulage en latex (x 1,5);6, même exemplaire, détail (x 2,1 env.); 7 , MT-225, moulage en latex (x 4); 8 , même exemplaire, détail (x 12 env.). 
gauche du ruisseau des Bonales; dans la partie supérieure des «Bancs Mixtes» (strates et alternances à Orthis auct.), dont l'âge correspond au Caradoc supérieur (Hammann, 1976; localité Al-I).

A suivre, les échantillons étudiés:

Al-IV: MT-208, 017, 018, 032 = Batalleria.

Al-VII: MT-228 = Destombesia. MT-223, 224, 225, 226, $227=$ Calix gutierrezi.

CC-IV: MT-210 (Phlyctocystis cf. Gigas) + MT-209 (Ph. granulata). MT-213 = Calix sp. aff. segaudi.

VM III: MT-211, 212 = Phlyctocistis cf. gigas.

\section{Paléontologie systematique}

CYSTOIDEA von Buch, 1846.

DIPLOPORITA Müller, 1854.

Famille Aristocystitidea Neumayr, 1889.

Genre Phlyctocystis Chauvel, 1966.

Phlyctocystis granulata Chauvel 1966 (espècetype).

Synonymie in Chauvel, 1978.

(P1. I, fig. 1).

Matériel.-Un exemplaire, MT-209, collection Gutiérrez Marcos.

Description.-Moule externe d'un fragment d'une thèque dont la forme et la taille sont inconnues (dimensions de l'empreinte: $85 \times 42 \mathrm{~mm}$ ). Plaques polygonales à surface externe régulièrement et plus ou moins convexe sans trace de tubercules-piquants, polygones très variés, de 5 à 9 côtés; on distingue des principales $\boldsymbol{P}$ dont; certaines sont presqu'aussi larges que hautes (ex. $8 \times 10,9 \times 11$ ), tandis que d'autres plus étroites atteignent $13 \mathrm{~mm}$, des intercalaires $i$ généralement pentagonales, plus larges que hautes $(6,5 \times 5)$ ou plus hautes que larges $(12 \times 6,5)$; la disposition parait irrégulière mais on reconnait des alignements transversaux d'intercalaires avec pentagonales tête..bêche. Fossettes diploporiques nombreuses, réparties sur toute la plaque, serrées, parfois subtangentes, elliptiques $(0,9 \times 0,5 ; 0,75 \times 0,6 \mathrm{~mm})$, sur les grandes plaques la plupart des fossettes ont leur grand axe dirigé vers le centre de la plaque, elles forment des alignements radiaux mais il y a des irrégularités assez nombreuses, sur les plaques petites ou allongées la disposition des fossettes est irrégulière; les fossettes sont ouvertes, cependant quelques-unes sont closes.

Observations.-La forme et la disposition des plaques rappellent plusieurs Aristocystitidés, ce n'est pas Aristocystites (dont les fossettes ne sont pas elliptiques) mais par contre cet exemplaire rappelle tout a fait l'holotype marocain et certains fossiles armoricains attribués à Phlyctocystis granulata (Chauvel, 1966; Pl. XII et Chauvel \& Nion, 1969), et bien que les bour- souflures du test ne soient pas visibles ayant été gommées à la fossilisation.

Repartition.-Ordovicien moyen. France (Massif armoricain), Espagne (Sierra Morena), Maroc (AntiAtlas).

Phlyctocystis cf. gigas (G. \& H. Termier, 1950).

Synonymie in Chauvel et Le Menn, 1979.

Matériel.-Trois exemplaires MT-210, 211 et 212, collection Gutiérrez Marcos.

Description.-Ce sont des fragments des moules internes de trois thèques qui ont été aplaties, les empreintes mesurent respectivement $66,68,94 \mathrm{~mm}$ de large ce qui correspond à des thèques qui, si elles étaient cylindriques, avaient de 40 à $60 \mathrm{~mm}$ de diamètre, mais leur forme exacte est inconnue (l'aplatissement sans pulvérisation laissant supposer que la section transversale était plutôt une ellipse très aplatie).

MT-211 montre une extrémité régulièrement arrondie les deux bords latéraux se rapprochant lègèrement dans l'autre sens, c'est donc une zone orale; sur MT210 l'extrémité est également arrondie mais les bords latéraux s'écartent nettement ce qui indique une extrémité aborale (cf. certains exemplaires marocains).

Les plaques sont nombreuses et petites. Sur MT212 la disposition en cercles réguliers est visible; quelques principales $P$ arrondies $(5$ à $7 \mathrm{~mm}$ ) ont jusqu'à huit côtés, les intercalaires sont variées et de divers ordres: 5 ou 6 côtés et aussi larges que hautes (ex: $4 \times 4,25), 5$ côtés plus hautes que larges et disposées tête-bêche, très petites, pentagonales ou triangulaires $(1,75$ ou $2 \mathrm{~mm})$; certaines principales sont entourées par un cercle d'intercalaires. Le moule interne des plaques est légèrement bombé (face interne concave). Les moules internes (dont la conservation est médiocre) portent des moulages de canaux thécaus souvent groupés par deux et surmontant des moulages de fossettes internes allongées; d'autres canaux proches des sutures sont coudés et s'appuient sur les cloisons interplaques (pôle aboral de MT-210); en outre de petites cassures et la fusion accidentelle de fossettes voisines de part et d'autre d'une suture peuvent avoir l'apparence de liaisons transsuturales rappelant les structures signalées chez Batalleria (voir plus loin).

Un fregment de moule externe sur MT-212, montre que la face externe des plaques est légèrement convexe, malgrè la mauvaise conservation on peut repérer quelques moulages de fossettes diploporiques (externes); elles sont allongées, nombreuses mais non serrées, orientées radialement (au moins sur les grandes plaques), on peut trouver sur MT-212 quelques très médiocres empreintes de pustules circulaires $(0,4$ à $0,5 \mathrm{~mm}$ ). Epaisseur du test: $1,5 \mathrm{~mm}$.

Observations.-L'existance possible de pustules (très médiocres), sur la face externe des plaques permet d'attribuer ce matériel à Phlyctocystis, la taille et la 
disposition des plaques, la forme supposée de la thèque plus particulièrement à $P$. gigas (détermination confirmée par une comparaison directe avec le matériel marocain; Chauvel, 1966, 1978). P. gigas a déja été signalé dans les Monts de Tolède (Chauvel et al., 1984), pour des exemplaires provenant de Navas de Estena et qui sont un peu différents du nouveau matériel (plaques intercalaires plus étroites, disposition des plaques plus régulière) ces différences ne sont probablement que des variations intraspécifiques, elles peuvent dépendre de la taille (donc de l'âge) des thèques et l'espèce n'est certainement pas exempte de polymorphisme.

Phlyctocystis cf. gigas est proche de Sinocystis Reed, de l'Ordovicien du Yunnan; les rapports des deux formes ont été précédement discutés (Chauvel, 1978).

Répartition.-Ordovicien moyen; France (Massif Armoricain), Espagne (Monts de Tolède, Sierra Morena), Maroc (Anti-Atlas).

Genre Calix Rouault, 1851.

Espèce-type: Calix sedgwicki Rouault, 1851.

Calix gutierrezi nov. sp.

(Pl. I, figs. 4-7; fig. in texte 1-2).

Matériel-Cinq échantillons: MT-123, 124, 125, 126 (holotype) et 127, collection Gutiérrez Marcos.

Locus-typicus.-Almadén, km 11, route d'Almadén à Saceruela.

Stratus-typicus.-Caradoc supérieur.

Derivatio nominis.-Espèce dédiée à J-C. Gutiérrez Marco.

Diagnose.-Une espèce de Calix dont les fossettes diploporiques sont polygonales.

Description.-MT-126 est un moule externe réduit à quatre éléments de cercles transversaux de plaques variées:

- Plaques principales P: huit côtes inégaux, bords latéraux parallèles, plaques plus hautes que larges (ex: $9,5 \times 8 \mathrm{~mm}$ ), nettement coniques, la base du cône couvrant toute la plaque, l'umbo souvent surélevé «en têton".

- Intercalaires i2 (?): semblables à il mais plus $9 \times 5,5 \mathrm{~mm})$, en position tête-bêche, coniques comme $P$.

- Intercalaires i2 (?): semblabes à il mais plus petites (ex. $7 \times 5 \mathrm{~mm}$ ), le cône parait plus pointu que sur P.

La succesion i-P-i est perturbée sur le côté de l'empreinte par suite de l'existence de plaques principales ou intercalaires irrégulières; certaines plaques sont seulement nettement convexes.

Les fossettes diploporiques (très nettes sur MT-224 et 225), couvrent entièrement la plaque, elles sont relativement grandes $(0,8$ à $1,1 \mathrm{~mm}$ env. $)$ et de for- mes variées, mais comme elles sont étroitement serrées cela leur donne une forme polygonale irregulière caractéristique et un moulage en latex montre un réseau à mailles polygonales. Il n'y a pas d'orientation particulière des fossettes; les canaux thécaux sont cylindriques $(0,2$ à $0,25 \mathrm{~mm})$.

Sur MT-224 l'umbo en teton se prolonge souvent en épine (tubercule-piquant); l'umbo, généralement central, peut être excentré sur certaines plaques. La forme générale de la thèque n'est pas connue, mais MT-224 permet de supposer une thèque allongée et
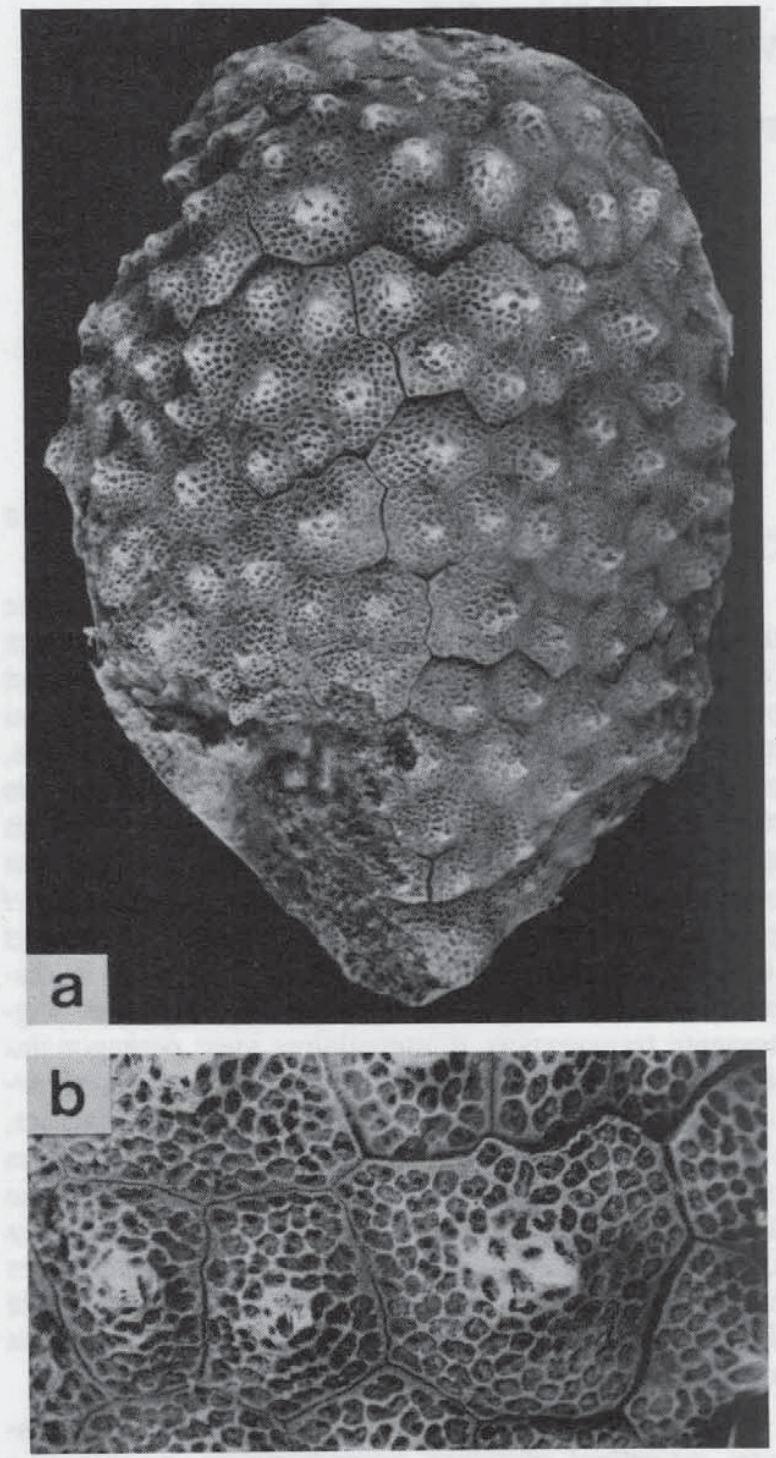

Fig. 1.-Calix gutierrezi n. sp.; paratype MT-227. Caradoc supérieur; Almadén (Ciudad Real). a) réplique en latex du moulage externe (x 1,3); b) détail du même moulage en latex, avec les fossetes diploporiques polygonales sur les plaques avec l'umbo «en teton", et quelques diplopores (x 3,7). Photos: E. Martin Castellanos. 
non globuleuse et une section transversale elliptique. Une succesion i2-il-i2-P, si fréquente chez les Diploporites est partiellement visible.

MT-227 est le moule externe assez grand $(80 \times 52 \mathrm{~mm})$, d'une thèque peut-être fusiforme; les plaques, disposées irregulièrement, de tailles et de formes très variées, sont fortement coniques; l'umbo de chaque plaque est nettement surélevé en pointe ou en têton; les fossettes diploporiques forment un réseau à mailles polygonales. A l'une des extrémités, une grande plaque avec fossettes de tailles variées, correspond probablement à la zone de fixation de la thèque; à l'autre extrémité, le péristome n'est pas conservé.

Observations.-L'existence de plaques coniques avec individualisation fréquente de tubercules en têtons justifie l'attribution au genre Calix. L'originalité de ce matériel résulte de l'aspect de ses fossettes diploporiques; les autres espèces de Calix ont des fossettes plus ou moins espacées: rondes ( $C$. rotundipora), elliptiques ( $C$. sedgwicki), allongées, vermiculaires ( $C$. rouaulti, segaudi...); l'espèce qui semble la plus proche de $C$. gutierrezi est $C$. termieri Chauv. 1966, mais si les plaques de $C$. termieri peuvent être côniques l'individualisation de tubercules-piquants est douteuse, si les fossettes diploporiques sont serrées elles le sont moins étroitement que chez $C$. gutierrezi si bien qu'elles sont allongées ou elliptiques plutôt que polygonales, si on compare les deux types on voit que les plaques de $C$. termieri sont en gros deux fois plus petites que celles de $C$. gutierrezi.

Des fossettes polygonales sont également visibles sur du matériel marocain attribué précédemment à $C$. sedgwicki (coll. Destombes IGR 16a, b \& 593a, in Chauvel 1966 p. 47-48, Pl. X, fig. 1,4); sur 16b deux plaques isolées portent des fossettes tout à fait comparables à celles de $C$. gutierrezi, sur 16a et 593a les fossettes sont moins nettes (sédiment grossier) et les plaques sont différentes de celles du Calix espagnol, (relief moins accentué), et leur gisement est plus ancien (Llandeilo).
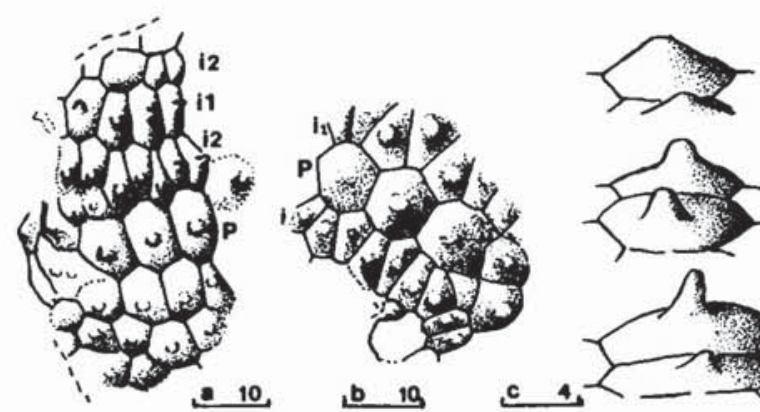

Fig. 2.-Calix gutierrezi n. sp. a) Exemplaire MT-224 qui montre les plaques principales $(P)$ et intercalaires $\left(i_{1}-i_{2}\right)$; b) exemplaire MT226. Sur a) et b), les fossetes diploporiques ne sont pas figurées. c) vues perspectives de quelques plaques: convexe, tubercule «en teton", tubercule-piquant. (les barres-échelle, en mm).
Des fossettes polygonales ont été signalées chez d'autres Diploporites très différents par ailleurs de $C$. gutierrezi par exemple: Tholocystis (Chauvel, 1941 et 1980, Pl. IV) et Sphaeronites (Peritaphros Paul, 1973), dont la thèque est globuleuse.

Les Calix son rares dans l'Ordovicien supérieur, $C$. hajraensis a été signalé à la base du Caradoc dans l'Anti-Atlas marocain (Chauvel et Destombes, 1978, p. 60), Calix sp. par Prokop dans le Caradoc de Bohême (1964, p. 15), Calix lebescontei Chauvel 1941, de l'Ordovicien supérieur du Massif armoricain est plus que douteux: des Calix ont été pris pour des Spongiaires, la confusion inverse n'est pas à exclure.

Calix sp. (aff, segaudi G. \& H. Termier 1950) (Pl. I, figs. 2, 3).

Matériel.-Un exemplaire MT-213, collection Gutiérrez Marcos.

Description.-Moule externe de la partie aborale d'une thèque dont l'extremité est rétrécie et déviée latéralement, la thèque s'évase largement mais de façon dissymétrique, on peut distinguer deux faces l'une étant étalée et peu convexe et l'autre redressée et nettement convexe (section transversale observée: $37 \times 31 \mathrm{~mm}$ ).

Les plaques, nombreuses et variées, sont disposées en cercles transversaux à peu près réguliers. On distingue quelques principales $\boldsymbol{P}$ isolées, à nombreux côtés (apparence subcirculaire, $\varnothing=5 \mathrm{~mm}$ ) et de nombreuses intercalaires de divers ordres:

- Polygonales arrondies à 8 côtés (ex.: $3,5 \times$ $\times 3,25 \mathrm{~mm})$.

- Hexagonales plus hautes que larges (ex.: $3,5 \times$ $\times 2,5 \mathrm{~mm}$ ).

- Pentagonales plus hautes que larges (ex.: $2,5 \times$ $\times 2 \mathrm{~mm}$ ) disposées tête-bèche.

- Pentagonales plus petites et plus larges que hautes (ex.: $1 \times 1,5 \mathrm{~mm})$, disposées tête-bèche.

Certains grandes plaques sont coniques et coiffées par un gros tubercule central dont la base n'occupe que le tiers de la plaque, il est à peu près aussi large que haut et son sommet est arrondi; sur d'autres plaques le tubercule n'est pas individualisé et la plaque est fortement conique. Les petites plaques sont plus ou moins convexes et on passe graduellement d'une forme a l'autre. Les fossettes diploporiques sont nombreuses mais non serrées, allongées (presque trois fois plus longues que larges et à bords parallèles) rectilignes, parfois légèrement arquées ou subelliptiques (variations dûes à la fossilisation), sur les grandes plaques leur orientation est radiale, moins régulière sur les petites plaques.

Observations.-MT-213 est une forme nouvelle mais qui doit être placée en nomenclature ouverte. Son aspect incite à le ranger dans le genre Calix qui 
est représenté dans les Monts de Tolède par plusieurs espèces à fossettes non elliptiques, le relief de ses plaques le rend proche particulièrement de Calix segaudi, espèce polymorphe dans laquelle on a distingué trois formes L, T, C, (Chauvel, 1978; p. 35, Pl. I, fig. 10), MT-213 pourrait être une forme T élargie. Sinocystis Reed a quelques plaques tuberculifères comparables à celles de MT-213 mais ses diplopores sont surélevés.

Famille Sphaeronitidae Neumayr, 1889.

Genre Batalleria Chauvel et Meléndez, 1978.

Diagnose (complétée).-Thèque oblongue, aux deux pôles semblablement arrondis, section transversale elliptique ou subcirculaire, nombreuses plaques de divers ordres, legèrement convexes, les plus grandes ne dépassant pas $7 \mathrm{~mm}$, fossettes diploporiques allongées à orientation généralement radiale, canaux tangentiels transsuturaux, appareil ambulacraire à cinq rayons courts avec bouquets brachiolifères.

Batalleria batalleri Chauvel et Meléndez 1978 (Pl. II, figs. 1, 2, 5, 6; figs. 2a, b in texte).
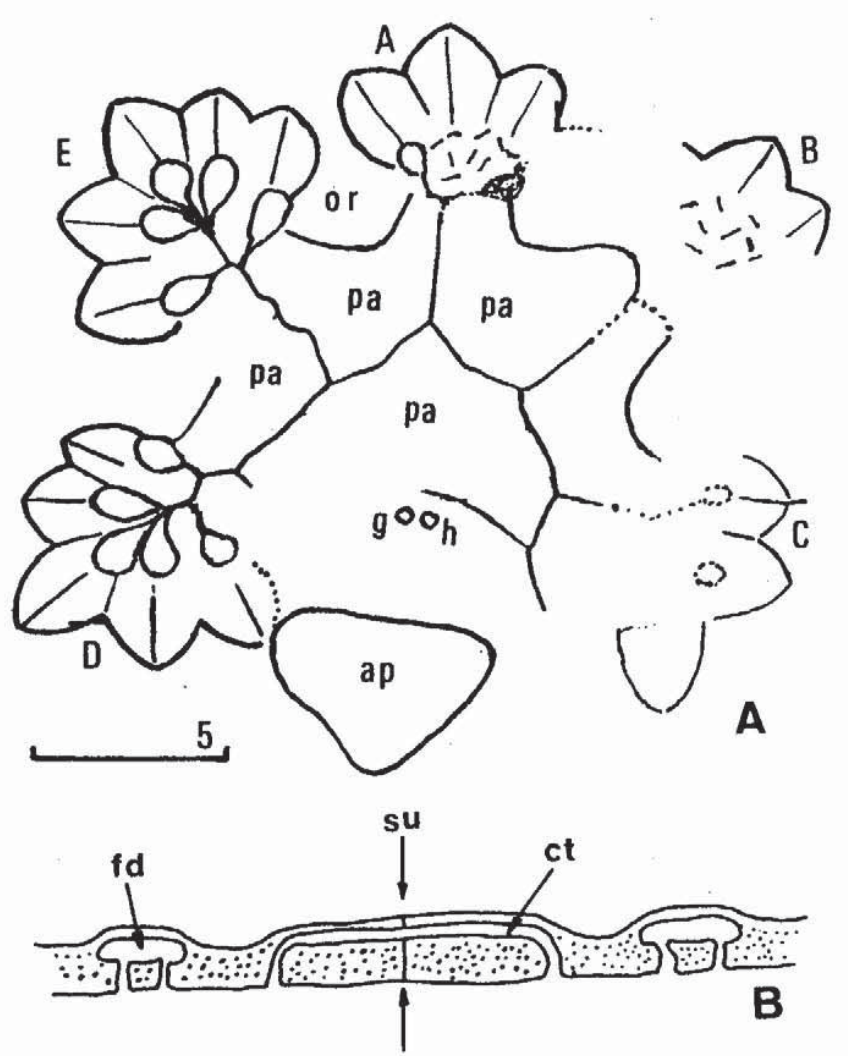

Fig. 3.-Batalleria batalleri, exemplaire MT-208: A) le póle adoral, rayons ambulacraires avec facettes brachiolifères (A...E), hydropore et gonopore $(h$ et $g$ ), emplacement de la pyramide anale $(a p)$, des plaques orales (or) et palatales ( $p a)$. B) Coupe schematique du test: fossette diploporique $(f d)$, suture entre deux plaques contigues $(s u)$, et canal transsutural $(c t)$.
1951. Echinosphaerites batalleri Meléndez, págs. 10-11; figs. 2, 4.

1978. Batalleria batalleri Chauvel et Meléndez, pags. 81-82; fig. 7 in texte; Pl. II, fig. 8.

Matériel.-Le matériel cité en 1978 (IGME 330; MT-017, 018, 032) a été revu et la diagnose complétée grâce á un nouvel échantillon, MT-208, provenant de la route d'Almadén à Ciudad Real, km 24; Llandeilo, collection Gutiérrez Marcos.

Description.-L'exemplaire MT-208 est un fragment de l'extrémité orale, exposant la surface externe de la thèque; les plaques sont petites, bombées, de divers ordres $(P \& i)$. Les fossettes diploporiques sont allongées, étroites, vermiculaires, parfois légèrement arqués, non serrées, orientées radialement (sauf sur quelques petites plaques); quelques rares fossettes se presentent en creux mais la plupart sont en relief ce qui suppose l'existence d'une couche mince recouvrant chaque fossette proprement dite. Des canaux transsuturaux rectilignes, également en relief, sont visibles entre les umbos de plaques voisines (mais sans les atteindre), il peut y avoir plusieurs canaux perpendiculaires à une suture mais pas de véritables rhombes; sur MT-017 ces canaux tangentiels débouchent à l'intérieur de la thèque; parfois des fossettes placées bout à bout peuvent être confondues avec des canaux tangentiels.

L'appareil ambulacraire est groupé (largeur: $22 \mathrm{~mm}$ env.) avec cinq rayons courts. L'ambulacre $E$ est le mieux conservé: il montre un double bouquet, le bouquet central a trois branches, la branche médiane est reliée distalement à trois empreintes ovales, allongées et légèrement surélevées, les deux branches latérales et proximales sont reliées à des ovales semblables. Cette partie centrale du bouquet est entourée par une corolle en éventail de cinq empreintes chacune correspondant à un ovale mais beaucoup plus grande, leur dessin est ogival, elles sont concaves et divisées en deux moitiés; l'ensemble correspond évidemment à l'insertion de cinq brachioles bisériés.

L'ambulacre D montre encore les cinq empreintes externes et le «bouquet central» mais les autres figures ambulacraires sont incomplètement conservées: trois (ou quatre) empreintes ogivales pour A, trois pour B et C.

Dans l'interradius CD et contre l'ambulacre $D$, un plateau un peu surélevé marque l'emplacement de la pyramide anale; il est triangulaire $(5,5 \times 4,5 \mathrm{~mm})$, avec les sommets arrondis, cette forme triangulaire peut paraitre inhabituelle: la pyramide anale des Cystoîdes étant souvent penta ou hexagonale, mais ce n'est pas une règle stricte. Dans l'interradius $C D$ et dans le prolongement du rayon $\mathrm{A}$ deux petites dépressions voisines et subcirculaires $(0,3$ à $0,4 \mathrm{~mm})$ sont l'hydropore et le gonopore, si elles ne sont pas accidentelles.

Les limites des plaques (parfois marquées sur le 

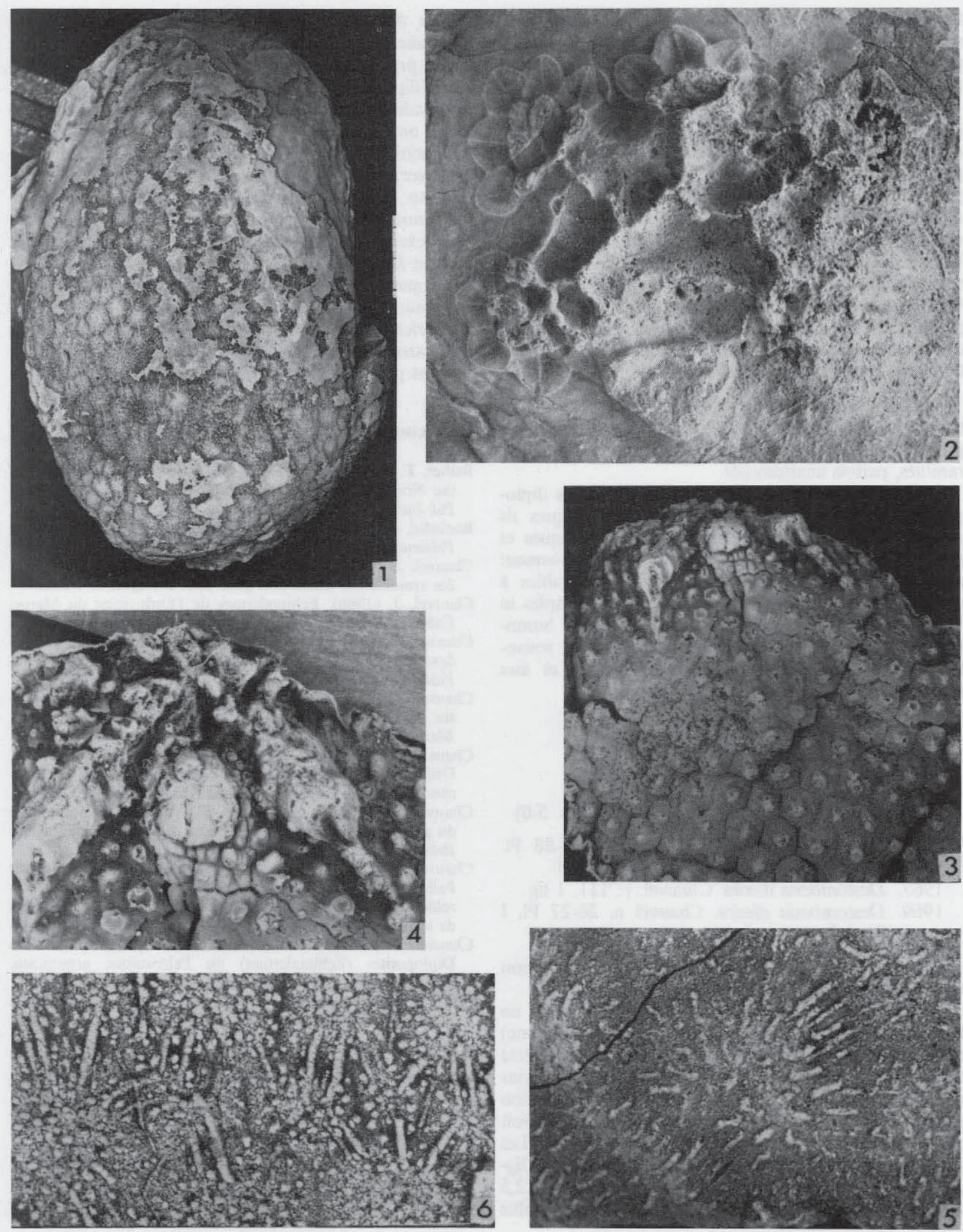

Planche II.-Figs. 1, 2, 5, 6: Batalleria batalleri: 1, holotype, . $^{\circ}$ 54-S IGME (x 1); 2, MT-208, pôle oral (x 4), cf. fig. 3 in texte; 5 , MT-208, détail de la surface externe de quelques plaques (x 9 env.); 6, MT-017, moule interne de quelques plaques (x 6). - Figs. 3, 4: Destombesia diedra MT-228: 3, moulage en latex (x 4); 4, détail du pôle oral (x 6). Photos: J. Chauvel, J. Vannier et D. Bernard. 
fossile par des crêtes plus ou moins accentuées), ne sont ni assez complètes ni assez nettes pour qu'on puisse décrire avec précision les plaques orales et palatales; il peut s'agir de fractures accidentelles.

Observations.-La description de 1978 confirmée et complétée par le nouveau matériel correspond à la diagnose des Sphaeronitidae telle qu'elle est proposée par le Treatise (S 241) l'incertitude concernant la position systématique de Batalleria est donc levée. Il se distingue des autres genres par sa forme générale oblongue; son appareil ambulacraire est aussi particulier avec ses facettes articulaires ogivales. Ses canaux transversaux (qui avaient pu faire croire à un Rhombifère), se retrouvent chez quelques Aristocystitidés; les plaques de ces derniers sont poreuses, traversées par des canaux divers (voir par exemple Bather, 1906; Chauvel, 1941, 1977; Bockelie, 1984):

- Les uns aboutissent à des fossettes diploporiques (parfois à des haplopores?): canaux simples, canaux groupés par deux, canaux en Y, canaux branchus, ramifiés, parfois anastomosés.

- Les autres sont indépendants des fossettes diploporiques partant de la face interne des plaques ils affrontent le plus souvent les cloisons interplaques et peuvent même traverser les sutures, ils deviennent ainsi des canaux tangentiels qui sont comparables à certains canaux de Rhombifères (fistulipores simples in Paul 1968, p. 699), mais en sont-ils vraiment homologues? Il n'est past impossible que ce soit un souvenir d'un ancêtre commun aux Diploporites et aux Rhombifères.

Famille Destombesiaidae Chauvel 1966.

Genre Destombesia Chauvel 1966.

Espèce-type: Destombesia diedra.

Destombesia diedra Chauvel 1966 (PI. II, figs. 5-6).

1966. Destombesia diedra. Chauvel pp. 83-88 Pl. V fig. 4, Pl. XIV fig. 7, PI. XVI fig. 1,3.

1967. Destombesia diedra. Chauvel, p. 111, 1 fig.

1969. Destombesia diedra. Chauvel p. 26-27 P1. I fig. 1-5.

Matériel--Un exemplaire MT-228, collection Gutiérrez Marcos; Almadén (C.R.); Caradoc.

Description.-C'est -dans un schiste ocreux - un fragment de thèque (moule interne et moule externe) qui malgrè son insuffisance montre bien les caractères de l'espèce. La thèque est oblongue (dimensions approximatives: $\mathbf{L}=35 \mathrm{~mm}, 1=25 \mathrm{~mm}$ ); la section transversale devait être pentagonale mais on ne voit qu'un pan encadré par deux pliures longitudinales. Les plaques sont nombreuses, disposées en mosaîque à lignes méridiennes, elles sont hexagonales, subégales $(2,5$ à $3 \mathrm{~mm}$ ) sur le pan latéral, mais deviennent plus petites dans la zone orale; chaque plaque porte un tubercule central cylindroconique dont la base occupe à peu près le tiers de la surface de la plaque; les fossettes diploporiques sont allongées, non serrées, disposées radialement. Le pôle oral est partiellement conservé, on reconnait la pyramide anale encadrée par les moignons fixés à la thèque $(*)$ des brachioles $\mathrm{C}$ et $\mathrm{D}$.

Observations. $-D$. diedra est une espèce polymorphe et plusieurs sous-espèces ont été distinguées mais notre exemplaire est trop incomplet pour être situé exactement, il semble proche du matériel décrit comme $D$. diedra subsp. (Chauvel, 1969).

Le genre Destombesia est connu dans l'Ordovicien supérieur (Ashgill et surtout Caradoc) du Massif Armoricain, d'Espagne (Asturies, Monts de Tolède) et du Maroc (Anti-Atlas), toutefois un gisement armoricain est peut-être plus ancien (Chauvel, 1967).

\section{Referencias}

Bather, F. A. (1906): In Reed: The Lower palaeoz. Fossils of the Northern Shan States Burma. Geol. Surv. India. Mem. Pal. Indica, 2, 154.

Bockelie, J. F. (1984): The Diploporita of the Oslo region. Palaeontology, 27, 1-68.

Chauvel, J. (1941): Recherches sur les Cystoides et les Carpoides armoricains. Mém. Soc. geol. min. Bretagne, 5, 1-286.

Chauvel, J. (1966): Echinodermes de l'Ordovicien du Maroc. Cahiers de Paléontologie, C.N.R.S., 112 p, París.

Chauvel, J. (1967): Présence du genre Destombesia dans l'Ordovicien du Massif armoricain. $C$. R. som. Soc. geol. France, 3, p. 111.

Chauvel, J. (1969): Données nouvelles sur le genre Destombesia de l'Ord. de l'Anti-Atlas marocain. Notes Serv. géol. Maroc, 29, 25-30.

Chauvel, J. (1977 a): Calix sedgwicki Rouault (Echin. Cyst. Ord. Massif armor.) et l'appareil ambulacraire des Diploporites. C. R. som. Soc. geol. France, 6, 314-317.

Chauvel, J. (1977 b): In Chauvel \& Truyols: Sur la présence du genre Destombesia dans l'Ordov. des Asturies (Espagne). Breviora Geologica Asturica, 21, 37-40.

Chauvel, J. (1978): Compléments sur les Echinodermes du Paléozoîque marocain (Diploporites, Eocrinoîdes, Edrioastéroîdes). Notes Serv. geol. Maroc, 39, 27-78 (avec un tableau de répartition stratigraphique par J. Destombes).

Chauvel, J. (1980): Données nouvelles sur quelques Cystoîdes Diploporites (Echinodermes) du Paléozoĩque armoricain. Bull. Soc. géol. min. Bretagne, 12, 1-18.

Chauvel, J. et Le Menn, J. (1979): Sur quelques Echinodermes (Cystoîdes et Crinoîdes) de l'Ashgill d'Aragon (Espagne). Geobios, 12, 549-587.

Chauvel, J. et Meléndez, B. (1978): Les Echinodermes (Cystốdes, Asterozoaires, Homalozoaires) de l'Ordov. moyen des Monts de Tolède (Espagne). Estudios geol., 34, 75-87.

Chauvel, J. et Nion, J. (1969): Sur la présence de Phlyctocystis (Echin. Cystoîde) dans l'Ordov. de la presqu'ile de Crozon. Bull. Soc. geol. France, 11, 443-446.

Gutiérrez Marco, J. C.; Chauvel, J.; Meléndez, B., et Smith, A. B. (1984): Los Equinodermos (Cystoidea, Homalozoa, Stelleroidea, Crinoidea), del Paleozoico inferior de los Montes de

(*) Structure qu'on pourrait peut-être rapprocher de ce que montre Eumorphocystis (suggestion de C. R. C. Paul). 
Toledo y Sierra Morena (España). Estudios geol., 40, 421-453.

Hammann, W. (1976): Trilobiten aus dem oberen Caradoc der östlichen Sierra Morena (Spanien). Senckenbergiana Lethea, 57, 35-85.

Kesling, R. V. (1967): Cystoids. in Treatise Invertebrate Paleontology (R. C. Moore ed.). Part S, Echinodermata, 85-267.

Meléndez, B. (1951): Sobre un notable cistideo del Silúrico español. Echinosphaerites murchisoni. Vern. y Barr. Libro Jubilar, 2. Instituto Geológico y Minero de Espafia. 1-15.

Paul, C. E. C. (1968): Morphology and function of dichoporite pore-tructures in cystoids. Palaeontology, 2, 697-730.

Paul, C. R. C. (1973): British Ordovician Cystoids Part 1, 164, Palaeontological Society monographs. London.

Recibido el 5 de noviembre de 1986 Aceptado el 11 de diciembre de 1986 